\title{
Different Kinetics of Oxidative Stress and Inflammatory Markers after Eccentric Exercise in Upper and Lower Limbs ${ }^{\dagger}$
}

\author{
Eleanna Chalari 1,*, Spyridon Methenitis ${ }^{2}$, Giannis Arnaoutis ${ }^{1}$, Ioanna Stergiou 1, \\ Christina Kampouropoulou ${ }^{1}$, Efstathios Karampelas ${ }^{1}$, Niki Prousinoudi ${ }^{1}$, \\ Vassiliki Argyropoulou ${ }^{1}$ and Tzortzis Nomikos ${ }^{1}$ \\ 1 Department of Nutrition and Dietetics, School of Health Sciences and Education, Harokopio University, \\ 17676 Athens, Greece \\ 2 Sports Performance Laboratory, School of Physical Education and Sports Science, National and \\ Kapodistrian University of Athens, 15772 Athens, Greece \\ * Correspondence: e.chalari@gmail.com \\ + Presented at the 9th Greek Conference of Biochemistry and Physiology of Exercise, Thessaloniki, Greece, \\ 18-20 October 2019.
}

Published: 2 September 2019

\begin{abstract}
AIM: Subclinical, low-grade, inflammation is one of the main pathophysiological mechanisms underlying the majority of chronic diseases. It is therefore obvious that an inflammatory model, inducing inflammatory responses to humans in a regulated, specific, and non-harmful way, could greatly facilitate the assessment of redox and immune status. Exercise-induced muscle damage (EIMD) could serve as such a model; however, the kinetics of biomarkers may be substantially different according to the muscle groups examined. Therefore, the aim of this study was to assess the responses of selected inflammatory and redox biomarkers after eccentric-induced muscle damage in upper and lower limbs. MATERIAL \& METHOD: Ten healthy, sedentary volunteers ( 5 males) performed, in a randomized and cross-over fashion, two discrete exercise protocols in lower $(10$ sets $\times 10$ drop jumps from a height of $40 \mathrm{~cm})$ and upper extremities ( 8 sets $\times 10$ eccentric muscle contractions of the biceps at $90 \%$ of $1 \mathrm{RM}$ ) separated by 6 weeks. Venous blood samples were collected pre-, immediately post-, 2, 24 and $48 \mathrm{~h}$ after the exercise protocols. Body composition was evaluated with dual-energy X-ray absorptiometry, blood cell count analysis by an automated hematology analyzer, CK, CRP and IL-6 by commercially available kits, while serum glutathione peroxidase (GPX3) by enzymatic assays adapted for microwell plates. RESULTS: A different pattern of response (time $\mathrm{x}$ protocol $p<0.05$ ) was observed for CK, IL-6 and CRP, with lower limbs reaching the highest increases at $2 \mathrm{~h}$ post-exercise, while upper limbs at 24 and $48 \mathrm{~h}$. A similar response between the two protocols was observed for WBC and neutrophils, with a transient $30 \%$ increase at $2 \mathrm{~h}$ (time $p<0.05$ ). No effect of time or protocol was observed for GPX3. CONCLUSIONS: Kinetics of selected inflammatory and redox biomarkers follow different patterns depending on the muscle group tested. Thus, depending on the targeted muscle group, blood samples should be obtained at specific time points. The higher levels of CK, IL-6 and CRP at 24-48 h implies an augmented inflammatory response at upper limbs, probably due to a lower adaptation to eccentric exercise, indicating that EIMD of upper limbs is a better protocol for the assessment of the inflammatory status in humans.
\end{abstract}

Keywords: eccentric exercise; inflammation; oxidative stress

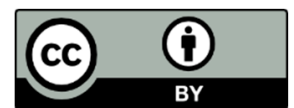

(C) 2019 by the authors. Licensee MDPI, Basel, Switzerland. This article is an open access article distributed under the terms and conditions of the Creative Commons Attribution (CC BY) license (http://creativecommons.org/licenses/by/4.0/). 